\title{
La milpa como alternativa de conversión agroecológica de sistemas agrícolas convencionales de frijol (Phaseolus vulgaris), en el municipio El Carmen de Viboral, Colombia
}

\author{
The milpa as a agricultural conversion alternative of conventional \\ agroecological systems of bean (Phaseolus vulgaris), in the municipality \\ of Carmen viboral, Colombia
}

\author{
Lina María Gómez Betancur ${ }^{1}$, Sara María Márquez Girón ${ }^{1}$, Luis Fernando Restrepo Betancur ${ }^{1}$
}

\section{RESUMEN}

El modelo de agricultura convencional en El Carmen de Viboral se fundamenta en un sistema de producción dependiente de un alto uso de insumos sintéticos, donde predomina el monocultivo, que se justifica como herramienta fundamental para lograr mayor eficiencia en el proceso productivo. Sin embargo, este sistema de producción ha mostrado serios problemas de sostenibilidad y ha ocasionado el deterioro de los recursos naturales. Por el contrario, la agroecología es una estrategia de desarrollo que se fundamenta en mejorar el suelo, fomentar el uso de insumos locales, dar mayor valor agregado y tener una cadena de comercialización más justa. Considerando la importancia que tiene el cultivo de frijol en El Carmen de Viboral, y la existencia de tres tipos de sistemas agrícolas para este producto en el municipio, se realiza un estudio del proceso de conversión de agricultura convencional a agroecológica, de forma participativa y fundamentado en la aplicación de principios ecológicos, transferidos de campesino a campesino.

La investigación se realizó de forma participativa con el propósito de elaborar una propuesta de diseño agroecológico y su implementación en fincas del municipio, complementado con el montaje de parcelas experimentales para evaluar el desempeño productivo del frijol. El tratamiento $\mathrm{N}^{\circ} 1$ fue monocultivo de frijol, el tratamiento $\mathrm{N}^{\circ} 2$ asociación de frijol con maíz sembrados con una diferencia de 45 días y el tratamiento $\mathrm{N}^{\circ} 3$ frijol y calabacín sembrados 45 días después de sembrar el maíz.

En la metodología estadística, se empleó un diseño de clasificación experimental en bloques aleatorizados efecto fijo balanceado, con tres réplicas por tratamiento. Se incorporó el análisis multidimensional de la varianza. Se complementó con la prueba de comparación de Tukey, análisis de componentes principales y proceso descriptivo unidimensional, a fin de establecer la media aritmética, la desviación típica y el coeficiente de variación.

Las variables a evaluar fueron: el número de vainas, peso, ancho y largo de la vaina, número de granos de frijol, peso y rendimiento. Al efectuar el análisis comparativo de los tratamientos se encontró diferencia estadística entre el tratamiento 3 y los tratamientos 1 y 2 para las variables número de vainas, peso de la vaina, número de frijoles, peso del frijol y rendimiento ( $\mathrm{p}<0,05)$. Se obtuvieron mejores resultados en el tratamiento 3. Para las variables ancho de la vaina y largo de la vaina, no se detectó diferencia estadística significativa ( $\mathrm{p}>0,05$ ). Se evaluó el uso equivalente de la tierra (UET) para los 3 tratamientos, encontrando el mejor resultado en la Milpa (Asociación frijol-maíz-calabacín).

Palabras clave: milpa, conversión, agroecología, policultivos, frijol, maíz, calabacín.

\begin{abstract}
The model of conventional agriculture in El Carmen de Viboral, is based on a production system dependent on a high use of synthetic inputs, where monoculture is justified as an essential tool for achieving greater efficiency in the production process. However, this production system has shown serious problems of sustainability and has caused the deterioration of natural resources. In contrast, agroecology is a development strategy that is based on improving the soil, encouraging the use of local inputs, giving greater added value and have a fairer marketing chain.

Considering the importance of the bean crop in El Carmen de Viboral, and the existence of three types of agricultural systems for this product in the municipality, a study of the conversion process from conventional agriculture to agro-ecological is carried out. This study was participatory and based on the application of ecological principles, transferred from farmer to farmer.

The research was conducted in a participatory manner in order to develop a proposal for agroecological design and its implementation on farms in the municipality, supplemented with the assembly of experimental plots to evaluate the productive performance of bean. Treatment No. 1 was monoculture beans, treatment $N^{\circ} 2$ was an association between bean and corn planted with a difference of 45 days and treatment $N^{\circ} 3$ beans and zucchini planted 45 days after sowing corn.
\end{abstract}

\footnotetext{
1 Universidad de Antioquia, integrante del grupo de investigación GISAS. Medellín, Colombia.

* Autor por correspondencia: lina.gomez6@udea.edu.co
} 
In statistical methodology, classification experimental design was used in randomized block balanced fixed effect, with three replicates per treatment. Incorporating multidimensional analysis of variance; it was complemented by the Tukey comparison test, principal component analysis and dimensional descriptive process to establish the arithmetic mean, standard deviation and coefficient of variation.

The variables evaluated were: the number of pods, weight, width and length of the pod, number of grains of beans, weight and performance. Carrying out the comparative analysis between treatments it was possible to observe statistical difference between treatment 3 showing better results regarding treatments 1 and 2, for the variables number of pods, weight of pod, number of beans, weight of beans and performance $(p<0,05)$. For variable width of the sheath and length of the sheath, no significant statistical difference $(p>0,05)$ was detected. the equivalent land use (UET) for 3 treatments was evaluated, finding the best result in the Milpa (Association bean-corn-zucchini).

Keywords: Milpa, Conversion, Agroecology, polyculture, beans, corn, zucchini.

\section{Introducción}

La transformación social en el municipio El Carmen de Viboral, Colombia, enlaza con el cambio agrícola de un modelo dominante y emancipado, vulnerable a plagas y enfermedades, y contaminante del medio ambiente, donde surge un grupo de productores abanderados de la conversión agroecológica, cuyo eje articulador es la familia campesina, y que aporta alternativas de manejo en el sistema productivo, con el fin de mejorar la sostenibilidad y la resiliencia de la finca.

En la transición de la agricultura de sistemas convencionales a sostenibles sobre bases agroecológicas, la biodiversidad constituye un recurso natural esencial que puede ser manejado por el agricultor, favoreciendo su conservación, así como la de los procesos ecosistémicos que contribuyen a la eficiencia del sistema de producción (Gliessman y Rosemeyer, 2010; Márquez, 2013; Vázquez et al. 2014; Sarandón y Flores, 2014).

El proceso se inicia con un cambio en la visión de los agricultores sobre sus fincas, con la decisión de trabajar procesos naturales y comprender que se trata de un sistema de producción nuevo y distinto al convencional, utilizando modelos que están diseñados para optimizar la salud del suelo y de los cultivos (Gliessman, 1998; Funes-Monzote, 2009).

La conversión de sistemas convencionales de producción, caracterizados por monocultivos manejados con altos insumos, a sistemas agroecológicos, se basa en la diversificación del hábitat y el manejo orgánico del suelo (Clavijo et al., 2006; Altieri y Nicholls, 2007).

En México, la agricultura campesina e indígena desde antes de la época de la colonia viene practicando el sistema de cultivo milpa, que integra al maíz, frijol, calabaza, chile y haba, en razón de que ha demostrado una alta capacidad de resistencia a condiciones adversas, así como de aseguramiento de la alimentación de las familias y de los animales, y el aumento de nutrientes de los suelos donde lo han implementado (Gutiérrez et al., 2007). El principal medio de difusión de este sistema ha sido la comunicación de campesino a campesino, lo que le ha permitido ser compartido con promotores de manera horizontal, revalorizando su alta diversidad y las propiedades de sus componentes alimentarios (Altieri, 1999; Gliessman, 2002; Clavijo, 2006).

Cada cultura, de acuerdo a sus saberes y tradiciones, selecciona las plantas y las combina de forma personal imprimiéndole a la milpa su sello particular, en la selección de variedades, manejo del sistema agrícola y procesamiento de productos, así como en la organización social en torno a los cultivos (Álvarez et al., 2011; Sánchez y Hernández, 2014).

En el sistema milpa se obtienen dos o tres cosechas que aventajan a lo que se lograría sembrando un solo cultivo. De esta manera, se optimiza el uso del suelo en las parcelas de los pequeños productores, y simultáneamente se producen más alimentos de calidad y libres de agroquímicos (Sánchez y Hernández, 2014).

Según Álvarez et al. (2011), la milpa es un policultivo agroecológico donde se conjugan conocimientos y tecnologías tradicionales haciendo uso eficiente de los recursos bióticos y abióticos de la naturaleza a lo largo del ciclo de cultivo, pues ha demostrado ser capaz de sustentar la alimentación sana y diversa de grandes poblaciones de manera sostenible, y donde la familia es el elemento más importante.

Una de las principales razones por las cuales los agricultores en el mundo adoptan los policultivos, en que en la mayoría de ellos se puede obtener un mayor rendimiento en un área sembrada como 
policultivo que de un área equivalente, sembrada en monocultivo. Lo anterior fue confirmado por Natarajan y Willey (1980, citados por Liebman en 1999), los cuales encontraron que 0,94 hectáreas de monocultivo de sorgo y 0,68 hectáreas de monocultivo de guandul fueron necesarias para producir las mismas cantidades de sorgo y guandul que se cosecharon en un policultivo de 1 hectárea. A esta relación se le conoce como el Coeficiente de tierra equivalente (UET o LER), es decir, un policultivo tiene un rendimiento mayor en un área determinada que el que puede tener como monocultivo. Todo lo anterior responde a que cuando dos plantas crecen juntas, es muy probable que se influyan mutuamente, bien sea directa o indirectamente, ya que cuentan con un espacio de influencia (Liebman, 1999).

El propósito de la presente investigación fue evaluar los resultados de un proceso de conversión agroecológica participativa del cultivo de frijol en una zona agrícola del municipio El Carmen de Viboral - Antioquia, donde se propone la estructuración y transición de sistemas de producción convencionales a sostenibles.

\section{Materiales y métodos}

La zona de estudio fue el municipio El Carmen de Viboral, localizado en la subregión Oriente del departamento de Antioquia, Colombia. Con una altitud de $2.150 \mathrm{msnm}$, cuenta con una extensión total de $448 \mathrm{~km}^{2}$, en el área urbana $2.44 \mathrm{~km}^{2} \mathrm{y}$ en la rural $445.56 \mathrm{~km}^{2}$. Tiene 46.751 habitantes según DANE, 2015, de los cuales 30.107 viven en la zona urbana y 16.453 en la zona rural, distribuidos en 55 veredas. Sus coordenadas son: 604'55'N 75²0'03'”.

El método de investigación que se utilizó corresponde a una Investigación Acción Participativa, ajustada y adaptada a las unidades del taller AIL (Apoyando Innovaciones Locales), aplicando además el enfoque de sistemas en las fincas de los productores. La investigación se inició conformando un grupo de 16 productores de diferentes veredas del municipio El Carmen de Viboral, con los que se realizaron visitas a fincas y con la ayuda del taller AIL modificado, que consistió en 7 etapas, se trazó un camino hasta llegar a la elaboración del diseño de conversión agroecológica de frijol, concertado con todos los participantes (Bruschweiler y Rist, 2005).

\section{Tratamientos para el cultivo de frijol}

Los tratamientos evaluados según validación previa con los agricultores fueron:

Tratamiento 1: Frijol en monocultivo orgánico. Tratamiento 2: Frijol sembrado 45 días después de sembrar el maíz.

Tratamiento 3: Frijol y calabacín sembrados 45 días después de sembrar el maíz.

Cada parcela estuvo compuesta de la siguiente manera:

- Ocho surcos de 18 plantas cada uno, para un total de 144 sitios por parcela.

- Distancias de siembra entre surcos 1 metro y 70 centímetros entre plantas.

- Tres repeticiones por cada uno de los tratamientos.

- Aplicación de Bocashi a razón de 4 toneladas por hectárea.

Los datos analizados fueron los relacionados con la parcela útil, compuesta por 30 plantas centrales en cada uno de los tratamientos y repeticiones.

El grupo de productores validó diferentes propuestas de prácticas agroecológicas para el diseño de conversión según las innovaciones aprendidas y compartidas, entre las que se encontraban: destrucción de socas y residuos vegetales del lote, preparación del terreno con labranza mínima, uso de policultivos con semillas sanas y productivas de frijol, maíz y calabacín, época de siembra apropiada, espaciamiento adecuado, rotación de cultivos, visitas periódicas al cultivo, desinfestación de herramientas, aplicación de materia orgánica, cobertura del suelo, recolección de plantas enfermas, eliminación de focos de infección, siembra de plantas hospederas de enemigos naturales, adecuado manejo de plagas por medio del control biológico y el uso de biopreparados y cosechas oportunas.

Luego de efectuar una forma participativa y partiendo del monocultivo, los productores eligieron el maíz y el calabacín como cultivos asociados al frijol, semejando una MILPA (Figura 1). Además fue seleccionada la biodiversidad funcional acompañante por los beneficios ecológicos (Tabla 1). Se sembró botón de oro en los límites laterales de cada parcela, caléndula en la parte superior y albahaca y cilantro intercalados en la parte inferior. 


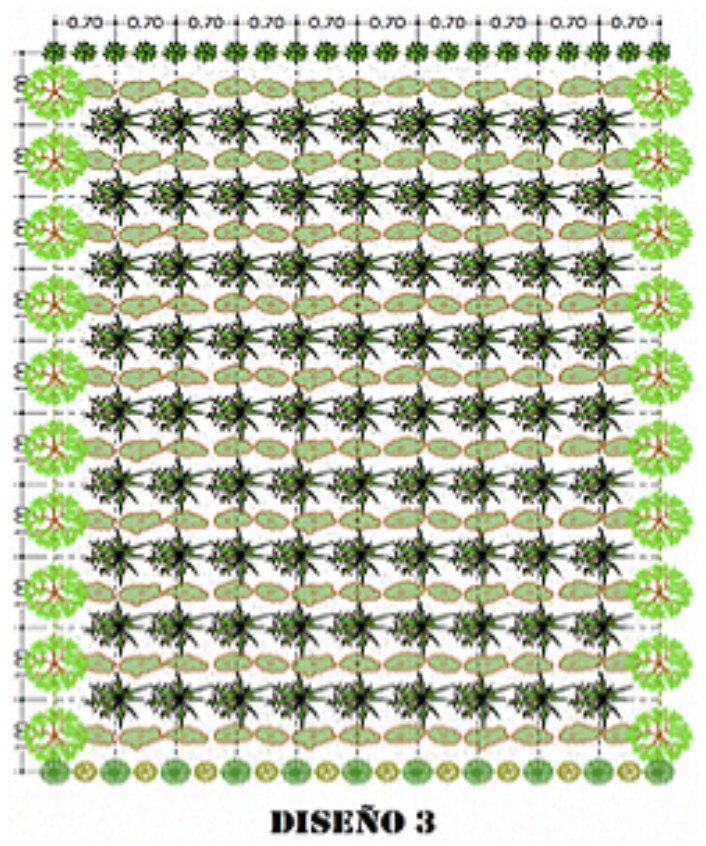

Figura 1. Diseño Agroecológico para la conversión de frijol.
Se determinó el uso equivalente de la tierra (UET) utilizando las siguientes fórmulas:

Frijol-Maíz-Calabacín

$$
\mathrm{UET}=\frac{\mathrm{Pf}}{\mathrm{Mf}}+\frac{\mathrm{Pm}}{\mathrm{Mm}}+\frac{\mathrm{Pc}}{\mathrm{Mc}}
$$

Para la asociación Frijol-Maíz

$$
\mathrm{UET}=\frac{\mathrm{Pf}}{\mathrm{Mf}}+\frac{\mathrm{Pm}}{\mathrm{Mm}}
$$

Donde:

Pf $=$ Rendimiento del frijol en policultivo.

Mf = Rendimiento del frijol en monocultivo.

$\mathrm{Pm}=$ Rendimiento del maíz en policultivo.

$\mathrm{Mm}=$ Rendimiento del maíz en monocultivo.

Pc $=$ Rendimiento del calabacín en policultivo.

$\mathrm{Mc}=$ Rendimiento del calabacín en monocultivo.

\begin{tabular}{|c|c|c|c|}
\hline Estrato & Especie & Distancias de siembra & Función agroecológica \\
\hline \multirow{4}{*}{ Bajo } & Calabacín & $\begin{array}{l}0,7 \mathrm{~m} \text { entre plantas y } 1 \mathrm{~m} \\
\text { entre surcos }\end{array}$ & $\begin{array}{l}\text { El calabacín es una hortaliza de porte rastrero. Este hábito de crecimiento } \\
\text { ayuda también como cobertura y protección del suelo. Pertenece a la } \\
\text { familia de las cucurbitáceas (Ott, 2009). }\end{array}$ \\
\hline & Cilantro & $10 \mathrm{~cm}$ entre plantas & Tiene acción insecticida y repelente. (Millán, 2008). \\
\hline & Caléndula & $10 \mathrm{~cm}$ entre plantas & $\begin{array}{l}\text { La caléndula atrae, debido al colorido de sus flores, insectos benéficos, } \\
\text { por lo que puede ser empleada en siembras asociadas; intercalada } \\
\text { con yerbabuena es excelente para el control de nematodos y moscas } \\
\text { blancas. (Millán, 2008). }\end{array}$ \\
\hline & Albahaca & $10 \mathrm{~cm}$ entre plantas & $\begin{array}{l}\text { Posee acción repelente, insecticida y acaricida. } \\
\text { Afecta a organismos tales como polillas, moscas, mosquitos, escarabajos, } \\
\text { pulgones, gusanos y ácaros. (Millán, 2008). }\end{array}$ \\
\hline \multirow{3}{*}{ Medio } & Frijol & $\begin{array}{l}0,70 \mathrm{~m} \text { entre plantas y } 1 \mathrm{~m} \\
\text { entre surcos }\end{array}$ & $\begin{array}{l}\text { Uno de los tres cultivos básicos de la milpa. Ofrece vainas tiernas } \\
\text { para consumo inmediato o semillas secas para su almacenamiento y } \\
\text { consumo posterior. Ayuda a fijar en el suelo el nitrógeno que el maíz } \\
\text { requiere para crecer (Muñoz Saldaña, 2010). }\end{array}$ \\
\hline & Maíz & $\begin{array}{l}0,70 \mathrm{~m} \text { entre plantas y } 1 \mathrm{~m} \\
\text { entre surcos }\end{array}$ & $\begin{array}{l}\text { Es un cereal importante que hace parte de los alimentos básicos en la } \\
\text { dieta del ser humano, sirve de soporte para el desarrollo y crecimiento } \\
\text { de frijol y corta ciclos de plagas. Se convierte además en buena fuente } \\
\text { de biomasa para abonos orgánicos (Serratos, 2009). }\end{array}$ \\
\hline & Botón de oro & $15 \mathrm{~cm}$ entre plantas & $\begin{array}{l}\text { Fuente de néctar para la alimentación de los enemigos naturales, forraje } \\
\text { de buen valor nutricional para la alimentación animal, capacidad de } \\
\text { restaurar suelos degradados, dinamizador de los ciclos de los nutrientes } \\
\text { en el suelo y repelente de insectos (Calle y Murgueitio, 2008). }\end{array}$ \\
\hline
\end{tabular}

Tabla 1. Cultivo principal, cultivos acompañantes y biodiversidad funcional. 


\section{Metodología estadística}

Se empleó un diseño de clasificación experimental en bloques aleatorizados efecto fijo balanceado, con tres réplicas por tratamiento. Se incorporó el análisis multidimensional de la varianza MANOVA con contraste canónico ortogonal, cuyo fin fue evaluar de manera conjunta las variables respuesta reportadas en la Tabla 2. Se complementó con la prueba unidimensional, de Tukey con base en un nivel de significancia del $5 \%$, análisis de componentes principales y aplicación del método descriptivo unidimensional cuyo objetivo fue establecer la media aritmética, la desviación típica y el coeficiente de variación. Se utilizó el paquete estadístico SAS UNIVERSITY EDITION. Nota: las variables número de vainas y número de frijol se transformaron para realizar el análisis de varianza con base en la familia BOXCOX. Los tratamientos se asignaron de manera aleatoria dentro de cada bloque.

\section{Modelo de clasificación experimental}

$$
\mathrm{Y}_{\mathrm{IJS}}=\mu+\mathrm{T}_{1}+\mathrm{B}_{\mathrm{J}}+\mathrm{E}_{\mathrm{S}(\mathrm{IJ})}
$$

$\mathrm{Y}_{\mathrm{IJS}}$ : corresponde a cada una de las variables respuesta.
Tabla 2. Variables respuesta utilizadas.

\begin{tabular}{lll}
\hline \multicolumn{1}{c}{ Variable } & \multicolumn{1}{c}{ Tipo de variable } & \multicolumn{1}{c}{ Medida } \\
\hline Número de vainas & Cuantitativa discreta & Conteo \\
Peso de la vaina & Cuantitativa continua & Gramos \\
Ancho de la vaina & Cuantitativa continua & Milímetros \\
Largo de la vaina & Cuantitativa continua & Milímetros \\
Número de frijoles & Cuantitativa discreta & Conteo \\
Peso del frijol & Cuantitativa continua & Gramos \\
Rendimiento & Cuantitativa continua & Kilogramos/Ha \\
\hline
\end{tabular}

$\mu \quad$ : efecto promedio del experimento.

$\mathrm{T}_{\mathrm{I}}$ : efecto del i-ésimo tratamiento: tratamiento 1- frijol solo, tratamiento 2- frijol y maíz juntos, tratamiento 3 - frijol, maíz y calabacín.

$\mathrm{B}_{\mathrm{J}} \quad$ : efecto del bloque.

$\mathrm{E}_{\mathrm{S}(\mathrm{IJ})}$ : error experimental.

\section{Resultados y discusión}

Al efectuar el análisis comparativo de los tratamientos se encontró diferencia estadística entre el tratamiento 3 (frijol, maíz y calabacín) y los tratamientos 1 (frijol solo) y 2 (frijol y maíz juntos) para las variables número de vainas, peso de la vaina, número de frijoles, peso del frijol y rendimiento $(\mathrm{p}<0,05)$. Para las variables ancho de la vaina y largo de la vaina no se detectó diferencia estadística $(\mathrm{p}>0,05)$. Ver Tabla 3.

Tabla 3. Análisis descriptivo y comparativo.

\begin{tabular}{|c|c|c|c|c|c|c|}
\hline & \multicolumn{2}{|c|}{ Tratamiento 1} & \multicolumn{2}{|c|}{ Tratamiento 2} & \multicolumn{2}{|c|}{ Tratamiento 3} \\
\hline & Media \pm Std & $\mathrm{Cv}$ & Media \pm Std & $\mathrm{Cv}$ & Media \pm Std & $\overline{\mathrm{Cv}}$ \\
\hline Vainas & $14,1 \pm 5,5 b$ & 39,0 & $15,1 \pm 5,9 b$ & 39,0 & $21,1 \pm 7,4 \mathrm{a}$ & $\overline{35,0}$ \\
\hline Peso Vaina & $162,2 \pm 74,7 b$ & 46,0 & $168,0 \pm 63,9 b$ & 38,0 & $253,4 \pm 109 a$ & 43,2 \\
\hline Ancho Vaina & $16,0 \pm 189,8 \mathrm{a}$ & 5,2 & $15,9 \pm 1,5 \mathrm{a}$ & 9,4 & $16,0 \pm 1,5 \mathrm{a}$ & 9,3 \\
\hline Largo Vaina & $148,9 \pm 17,2 \mathrm{a}$ & 11,5 & $148,9 \pm 13,8 \mathrm{a}$ & 9,2 & $149,7 \pm 13,3 a$ & 8,8 \\
\hline \# de Frijoles & $62,1 \pm 27,0 b$ & 43,4 & $66,6 \pm 27,7 b$ & 41,5 & $96,5 \pm 40,9 \mathrm{a}$ & 42,3 \\
\hline Peso Fruto & $73,5 \pm 36,7 b$ & 49,9 & $81,9 \pm 38,3 b$ & 46,7 & $126,1 \pm 56,5 \mathrm{a}$ & 44,8 \\
\hline \multirow[t]{3}{*}{ Rendimiento } & $1050 \pm 524 \mathrm{~b}$ & 49,9 & $1170 \pm 548 \mathrm{~b}$ & 46,8 & $1801 \pm 808 \mathrm{a}$ & 44,8 \\
\hline & \multicolumn{6}{|c|}{ MANOVA } \\
\hline & \multicolumn{2}{|l|}{ Valor } & \multicolumn{2}{|l|}{$\mathrm{F}$} & \multicolumn{2}{|l|}{ Valor $\mathrm{p}$} \\
\hline Wilks' Lambda & \multicolumn{2}{|l|}{0,71} & \multicolumn{2}{|l|}{7,85} & \multicolumn{2}{|l|}{$<, 0001$} \\
\hline Pillai's Trace & \multicolumn{2}{|l|}{0,28} & \multicolumn{2}{|l|}{7,32} & \multicolumn{2}{|l|}{$<, 0001$} \\
\hline Hotelling-Lawley Trace & \multicolumn{2}{|l|}{0,38} & \multicolumn{2}{|l|}{8,39} & \multicolumn{2}{|l|}{$<, 0001$} \\
\hline Roy's Greatest Root & \multicolumn{2}{|l|}{0,36} & \multicolumn{2}{|l|}{16,0} & \multicolumn{2}{|l|}{$<, 0001$} \\
\hline & \multicolumn{2}{|l|}{ Valor propio } & \multicolumn{4}{|c|}{ Valor $\mathrm{p}$} \\
\hline Dimensionalidad 1 & \multirow{2}{*}{\multicolumn{2}{|c|}{$\begin{array}{l}0,3538 \\
0,0158 \\
\end{array}$}} & \multicolumn{4}{|c|}{$<0,0001$} \\
\hline Dimensionalidad 2 & & & \multicolumn{4}{|c|}{0,3898} \\
\hline \multicolumn{7}{|c|}{ Comparación canónica } \\
\hline Tratamiento 1 & \multicolumn{2}{|l|}{ Tratamiento 2} & \multicolumn{4}{|c|}{ Tratamiento 3} \\
\hline $\mathrm{c}$ & \multicolumn{2}{|l|}{$\mathrm{b}$} & \multicolumn{4}{|c|}{$\mathrm{A}$} \\
\hline
\end{tabular}


El análisis multivariado de la varianza MANOVA, en el cual se estudian de manera simultánea todas las variables respuesta, permitió detectar diferencias altamente significativas entre el tratamiento 3 y los tratamientos 1 y 2 ( $p<0,0001)$, como se aprecia en la Tabla 3. Cabe anotar que al realizar la prueba de dimensionalidad esta fue altamente significativa en la dimensión uno $(\mathrm{p}<0,0001)$.

El análisis de componentes principales fue muy similar para los tres tratamientos, donde el tratamiento 1 fue explicado en su variabilidad total en un $80,08 \%$, el tratamiento 2 en $87,08 \%$ y el tratamiento 3 en un $86,62 \%$, como se describe en la Tabla 4.

En la Figura 2, se evidencia un comportamiento heterogéneo de la precipitación durante el ciclo del cultivo, mostrando stress hídrico por falta de agua durante la etapa de siembra en los meses de marzo-abril y estrés hídrico por exceso de agua durante las últimas etapas hasta cosecha en los meses de septiembre-octubre. Esta situación se vio reflejada en el desempeño productivo de los tratamientos, cuando obtuvo mejores resultados el tratamiento 3, es decir, la milpa, donde la asociación de frijol, maíz y calabacín generó microclima de protección y tolerancia a los extremos climáticos soportados durante el desarrollo del cultivo.

\section{Ler o uet (uso equivalente de la tierra)}

La Figura 3 y la Tabla 5 muestran los resultados del LER en cada uno de los tratamientos evaluados, obteniendo la milpa (frijol, maíz y calabacín) los niveles más altos en el uso eficiente del suelo, con 2,95 y seguido por la asociación de Frijol y Maíz con 1,82. Ambos tratamientos fueron comparados con el rendimiento del monocultivo.

Estos resultados confirman lo encontrado por Liebman (1999), quien menciona que cuando dos plantas crecen juntas, se influyen mutuamente, bien sea directa o indirectamente, ya que cuentan con un espacio de influencia, La principal característica de la asociación maíz-frijol es que, si bien los rendimientos de cada especie son menores que los logrados en los correspondientes monocultivos, la combinación de ambos rendimientos es mayor por unidad de área, siendo más eficiente en el uso de recursos económicos y ambientales (agua, nutrientes y luz), al igual que en mano de obra y tierra (Gutiérrez et al. 2007). Lo que se refleja en el bienestar de las familias campesinas, al hacer un

Tabla 4. Análisis de componentes principales por tratamiento.

\begin{tabular}{ccccccccr}
\hline & \multicolumn{2}{c}{ Tratamiento 1 } & & \multicolumn{2}{c}{ Tratamiento 2 } & & \multicolumn{2}{c}{ Tratamiento 3 } \\
\cline { 2 - 3 } & Factor 1 & Factor 2 & & Factor 1 & Factor 2 & & Factor 1 & Factor 2 \\
\hline A & 0,91746 & $-0,0145$ & & 0,94172 & $-0,01811$ & & 0,95295 & $-0,09698$ \\
B & 0,92665 & 0,1422 & & 0,91616 & 0,17793 & & 0,96244 & 0,04325 \\
C & $-0,1567$ & 0,8162 & & $-0,39163$ & 0,75081 & & 0,07171 & 0,84354 \\
D & 0,27967 & 0,8944 & & 0,03727 & 0,87666 & & 0,32799 & 0,70560 \\
E & 0,95971 & 0,0032 & & 0,96800 & 0,00517 & & 0,97145 & $-0,08438$ \\
F & 0,97100 & 0,0421 & & 0,97650 & 0,05653 & & 0,98269 & $-0,08098$ \\
G & 0,97100 & 0,0421 & & 0,97650 & 0,05653 & & 0,98269 & $-0,08098$ \\
VP & 0,6586 & 0,1422 & & 0,6750 & 0,1958 & & 0,6889 & 0,1773 \\
\hline$\%$ & 0,8008 & & \multicolumn{2}{c}{0,8708} & & & 0,8662 \\
\hline
\end{tabular}

Número de vainas $=\mathrm{A} ;$ Peso vaina $=\mathrm{B} ;$ Ancho vaina $=\mathrm{C} ;$ Largo vaina $=\mathrm{D} ;$ Número de frijol $=\mathrm{E} ;$ Peso Frijol $=\mathrm{F} ;$ Rendimiento $=\mathrm{F} ; \mathrm{VP}=$ Indica valor propio $\%$ = Indica el porcentaje de la variabilidad total explicado.

\begin{tabular}{|c|c|c|c|c|c|c|c|c|c|c|c|c|}
\hline Mes & 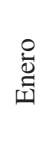 & $\begin{array}{l}\stackrel{0}{0} \\
\frac{0}{0} \\
\text { [I }\end{array}$ & $\frac{\stackrel{N}{\tilde{N}}}{\sum}$ & 泀 & 虫 & $\stackrel{\circ}{\Xi}$ & $\stackrel{\ominus}{\Xi}$ & 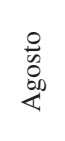 & 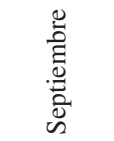 & 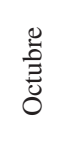 & 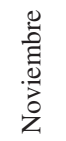 & $\begin{array}{l}\frac{0}{\tilde{U}} \\
\frac{0}{0} \\
\frac{0}{0}\end{array}$ \\
\hline Precipitación & 69 & 147 & 147 & 151 & 276 & 266 & 183 & 358 & 409 & 387 & 121 & 68 \\
\hline Etapa del cultivo & & & Siembra & & & & & & Cosecha & & & \\
\hline
\end{tabular}




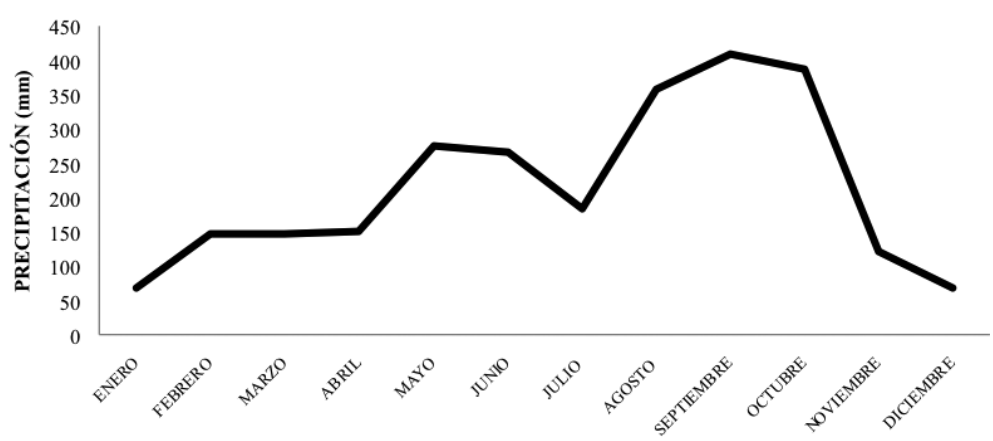

Figura 2. Comportamiento de la precipitación durante el ciclo del cultivo en el 2014.

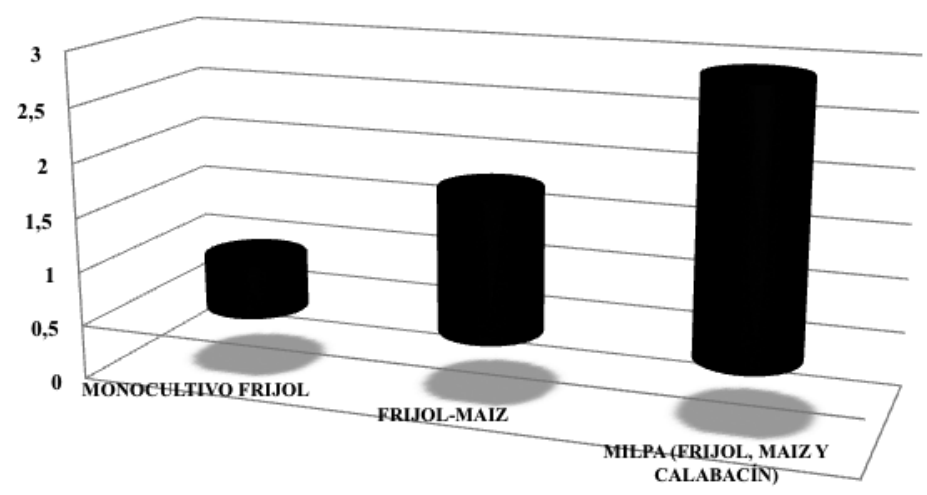

Figura 3. Gráfica del coeficiente del uso equivalente de la tierra LER o UET.

Tabla 5: Comportamiento del LER en cada uno de los tratamientos evaluados.

\begin{tabular}{lcccc}
\hline \multicolumn{1}{c}{ Tipo de cultivo } & Pf/Mf & Pm/Mm & Pc/Mc & LER \\
\hline Monocultivo frijol & 1,05 & & & 1 \\
Frijol-maíz & 1,11 & 0,708 & & 1,82 \\
Milpa (frijol, maíz y calabacín) & 1,71 & 0,966 & 0,27 & 2,95 \\
\hline Mf $=$ Rendimiento del frijol en monocultivo & & & & \\
Pm $=$ Rendimiento del maíz en policultivo & & & & \\
Mm $=$ Rendimiento del maíz en monocultivo & & & & \\
$\mathrm{Pc}=$ Rendimiento del calabacín en policultivo & & & & \\
$\mathrm{Mc}=$ Rendimiento del calabacín en monocultivo & & & & \\
\hline
\end{tabular}

mejor uso de su tierra, aprovechando los espacios, y obteniendo estabilidad ecológica y energética con incremento de la productividad.

Esta asociación con el calabacín permite mostrar los servicios ecosistémicos entre las tres especies con manejo agroecológico, llevando a fortalecer la biodiversidad y la seguridad alimentaria, con el uso de recursos locales que dan estabilidad a los sistemas, donde se incorpora el conocimiento tradicional coligado con la ciencia moderna (Oliveira, et al. 2014; Altieri y Nicholls, 2004). De esta manera se direcciona el pensamiento crítico a lo ecológico, a la conservación de los recursos que se tienen y se aprovechan mediante el uso de tecnologías respetuosas con la naturaleza, buscando ante todo el equilibrio ambiental (Altieri y Francis, 1992).

Por lo anterior, al promover nuevas tecnologías y prácticas, la agroecología optimiza los componentes biológicos y tecnológicos de los agroecosistemas, 
fundamentada en principios ecológicos y reconociendo el papel de los productores y extensionistas en la participación activa de redes de aprendizaje social (Marco, et al. 2003, Vázquez et al. 2008), facilitando la investigación, a partir de miradas desde la academia, la estadística, la ecología y sociología, evaluando la viabilidad técnica de los tratamientos de asociación para los cultivos frijol y maíz, que han sido implementados de forma tradicional por varios agricultores y transmitidos por herencia familiar o de campesino a campesino.

La asociación frijol, maíz y calabacín es ventajosa y adecuada por la facilitación que presentan los 3 cultivos. El maíz sirve como soporte para frijol voluble, contribuye a generar un microclima en tiempos de cambio climático, es barrera física, sitio de refugio de insectos benéficos y fomenta el manejo ecológico de arvenses. El frijol como leguminosa beneficia al maíz y al calabacín con la fijación de nitrógeno al suelo, y además genera un microclima que conserva la humedad en el sistema en momentos de estrés hídrico por sequía como se observó en la siembra, tal como lo menciona Toalá (2009). Otra ventaja importante es que los cultivos se pueden proteger entre sí de condiciones meteorológicas adversas y el calabacín ayuda como cobertura de suelo, disminuyendo mano de obra en desyerbas; cubre y protege el suelo y corta ciclos de plagas para el maíz y el frijol.

Además, una de las principales razones por las cuales los agricultores adoptan los policultivos, es que se puede obtener un mayor rendimiento en un área sembrada como policultivo que en un área equivalente, sembrada en monocultivo (Natarajan y Willey, 1980, citado por Liebman, 1999).

\section{Conclusiones}

Desde tempranas etapas del cultivo y hasta la cosecha, se evidenciaron diferencias significativas entre los tres tratamientos, respecto a las variables número de vainas, peso de vainas, número de granos y peso de grano, obteniéndose mejores resultados en el tratamiento de asociación $\mathrm{N}^{\circ} 3$ (siembra de maíz 45 días antes de la siembra del fríjol y el calabacín). Esto demuestra los beneficios de la interacción que tienen el frijol, el calabacín y el maíz en asociación, ya que el frijol fija nitrógeno en el suelo, el calabacín cubre el suelo y lo protege de efectos climáticos y de la erosión, y el maíz proporciona soporte y corta ciclos de plagas y enfermedades, lo que facilita el manejo del sistema productivo con mejores resultados.

Es importante relacionar el comportamiento de factores climáticos como la precipitación con el ciclo del cultivo, ya que se logró demostrar que aunque en el ciclo de cultivo los tratamientos tuvieron estrés hídrico por sequía durante la primera etapa, es decir, la siembra, y además soportaron estrés hídrico por inundación en la etapa de cosecha, el policultivo frijol-maíz y calabacín evidenció mayor tolerancia al generarse un microclima favorable por conservar la humedad en el suelo, evitando el marchitamiento por deshidratación y una afectación negativa por inundación, aprovechando el recurso presente.

Los resultados del LER en cada uno de los tratamientos evaluados mostraron a la milpa (frijol, maíz y calabacín) con los niveles más altos en el uso eficiente del suelo, con 2,95, seguido por la asociación de frijol y maíz con 1,82 . Ambos tratamientos fueron comparados con el rendimiento del monocultivo. Se confirmó que cuando dos plantas crecen juntas, se influyen mutuamente, y la combinación de cultivos lleva a mejores rendimientos, al ser más eficiente en el uso de recursos económicos y ambientales (agua, nutrientes y luz), lo que se refleja en el bienestar de las familias campesinas. Esta asociación con el calabacín permitió mostrar los servicios ecosistémicos entre las tres especies con manejo agroecológico, al fortalecer y aumentar la biodiversidad y la seguridad alimentaria, con el uso de recursos locales, donde se incorporó el conocimiento tradicional junto con la ciencia moderna.

\section{Literatura Citada}

Altieri, M.

1999. Agroecología: el camino hacia una agricultura sustentable. Capítulo 2: principios y estrategias para diseñar sistemas agrarios sustentables. Ediciones Científicas Americanas. pp. 27-34.
Altieri, M.; Francis, C.

1992. Incorporating agroecology into the conventional agricultural curriculum. American Journal of Alternative Agriculture, 7 (SpecialIssue 1-2): 93. 
Altieri, M.; Nicholls, C.

2004. Una base agroecológica para el diseño de sistemas diversificados de cultivo en el trópico. Revista: Manejo Integrado de Plagas y Agroecología. Costa Rica, 73: 8-20.

Altieri, M.; Nicholls, C.

2007. Conversión agroecológica de sistemas convencionales de producción: Teoría, estrategias y evaluación. En: Ecosistemas. 16 (1). Universidad de California. Berkeley, $10 \mathrm{p}$.

Álvarez, E.; Carreón, A.; San Vicente, A.

2011. Haciendo Milpa: La protección de las semillas y de la agricultura campesina. México, 104 p.

Brüschweiler, S.; Rist S.

2005. AIL - Apoyando Innovaciones Locales. Un instrumento para el fortalecimiento de innovaciones locales y la promoción del desarrollo rural sostenible. Perú.

Calle, Z.; Murgueitio, E.

2008. El botón de oro: Arbusto de gran utilidad para sistemas ganaderos de tierra caliente y de montaña. Carta FEDEGAN, 108: 54-63.

Clavijo, N.; Prins, C.; Sánchez, V.; Soto, G.; Staver, CH.

2006. Calendarización, uso racional, sustitución y rediseño: Una comparación entre horticultores orgánicos y convencionales en Costa Rica. Revista: Manejo Integrado de Plagas y Agroecología. Número 78: 17-27.

Funes-Monzote, F.

2009. Eficiencia energética en sistemas agropecuarios. Elementos teóricos y prácticos para el cálculo y análisis integrado. Indio Hatuey: Asociación Cubana de Técnicos Agrícolas y Forestales (ACTAF).

Gliessman, S.

1998. Agroecology: Ecological process in sustainable Agriculture, Ann Arbor Press. Turrialba, Costa Rica. CATIE, $359 \mathrm{p}$.

Gliessman, $\mathrm{S}$.

2002. Agroecología. Procesos ecológicos en Agricultura Sostenible. Turrialba, Costa Rica. CATIE, 359 p.

Gliessman, S.; Rosemeyer, M.

2010. The Conversión to sustainable Agriculture. Principles, processes and practices. CRC Press. United States of America, $352 \mathrm{p}$.

Gutiérrez, Z.; Aguilar, J.; Galdámez, J.; Mendoza, S.; Martínez, F. 2007. Impacto socioeconómico de los sistemas de policultivos maíz-frijol-calabaza en La Frailesca, Chiapas, México. En: I Seminario de cooperación y desarrollo en espacios rurales iberoamericanos sostenibles e indicadores. Almería, España, 16 p.
Liebman, M.

1999. Sistemas de Policultivos. Capítulo 9. En: Altieri. M. Agroecología, Bases científicas para una agricultura sustentable. Montevideo: Nordan-comunidad, Ecoteca 20.

Marco, O.; Reyes, R.

2003. Tecnologías limpias aplicadas a la agricultura. INCI. 5 (28). Caracas, Venezuela, 259 p.

Márquez, S.

2013. Agroecología y resiliencia socioecológica: adaptándose al cambio climático. Propuesta de conversión agroecológica para alcanzar la resiliencia en sistemas ganaderos. REDAGRES, SOCLA, Universidad de Antioquia, Universidad Nacional de Colombia, CYTED. Colombia. pp. 158-180.

Millán, C.

2008. Las plantas una opción saludable para el control de plagas. Red de acción en plaguicidas y sus alternativas para América Latina, Global Greengrants Fund. ISBN: 978-9974-8029-2-6. Impreso en I. Rosgal S.A., 101 p.

Oliveira, E.; Andrade, F.; Mello, J.; Machado, T.; Pereira, C.

2014. Efficiency assessment for a group of agroecological family farmersusing data envelopment analysis. Horticultura Brasileira, 3 (32).

Ott, S.

2010. Manual de cultivo de hortalizas. Cómo cultivar y cosechar las hortalizas de su jardín o su huerto. Ediciones OMEGA, $187 \mathrm{p}$.

Sánchez, P.; Hernández, P.

2014. Sistema Milpa: Elemento de identidad campesina e indígena. PIDAASSA. México, 26 p.

Sarandón, S.; Flores, C.

2014. Agroecología: Bases teóricas para el diseño y manejo de agroecosistemas sustentables. $1^{\text {a }}$ Ed. La Plata. Universidad Nacional de La Plata, 466 p.

Serratos, J.

2009. El origen y la diversidad del maíz en el continente americano.Universidad Autónoma de la Ciudad de México, $36 \mathrm{p}$.

Vásquez, L.; Fernández, E.; Alfonso, J.

2008. Impactos de las innovaciones tecnológicas realizadas por los propios agricultores sobre el manejo agroecológico de plagas en fincas de la agricultura urbana. Disponible en: http:www.socla.org/publicaciones. Consultado: 01/Ene/2017. Vázquez, L.; Matienzo, Y.; Griffon, D.

2014. Diagnóstico participativo de la biodiversidad en fincas en transición agroecológica. La Habana, Cuba, 3 (18): 151-162 p. 
